Lee Tuo-Yeong, Mathematics and Mathematics Education Academic Group, National Institute of Education, Nanyang Technological University, 1

Nanyang Walk, Singapore 637616, Republic of Singapore. e-mail:

tylee@nie.edu.sg

\title{
THE SHARP RIESZ-TYPE DEFINITION FOR THE HENSTOCK-KURZWEIL INTEGRAL
}

\begin{abstract}
In this paper, we prove that if $f$ is Henstock-Kurzweil integrable on a compact subinterval $[a, b]$ of the real line, then the following conditions are satisfied: (i) there exists an increasing sequence $\left\{X_{n}\right\}$ of closed sets whose union is $[a, b]$; (ii) $\left\{f \chi_{X_{n}}\right\}$ is a sequence of Lebesgue integrable functions on $[a, b]$; (iii) the sequence $\left\{f \chi_{X_{n}}\right\}$ is Henstock-Kurzweil equiintegrable on $[a, b]$. Subsequently, we deduce that the gauge function in the definition of the Henstock-Kurzweil integral can be chosen to be measurable, and an indefinite Henstock-Kurzweil integral generates a sequence of uniformly absolutely continuous finite variational measures.
\end{abstract}

\section{Introduction}

E. J. McShane in [9] developed the Lebesgue integration on an interval $I \subset \mathbb{R}^{n}$ using the monotone convergence of step functions. In dimension one, it is well-known (see [5] or [6]) that if $f$ is Denjoy-Perron integrable on a compact subinterval $[a, b]$ of the real line $\mathbb{R}$, then it can be defined as a controlled convergent sequence of step functions. Since the Controlled Convergence Theorem is equivalent to the equi-integrability theorem (see [4, Theorem 5.4]), it is natural to ask the following question : given that $f$ is Henstock-Kurzweil integrable on $[a, b]$, can $[a, b]$ be decomposed into a countable union of closed sets $\left\{X_{n}\right\}$ so that for each $n, f \chi_{x_{n}}$ is Lebesgue integrable on $[a, b]$, and $\left\{f \chi_{x_{n}}\right\}$ is Henstock-Kurzweil equi-integrable on $[a, b]$ ? In this paper, we shall give an affirmative answer to the above problem (see Theorem 3.7). The importance of this equi-integrability theorem lies in the construction of a topology $\mathcal{J}$ on

Key Words: Henstock-Kurzweil integral, equi-integrability

Mathematical Reviews subject classification: 26A39

Received by the editors November 11, 2001 
the space $\mathcal{H} \mathcal{K}([a, b])$, namely the space of all Henstock-Kurzweil integrable functions on $[a, b]$, so that the resulting space $(\mathcal{H} \mathcal{K}([a, b]), \mathcal{J})$ is complete. See [3] for more details. Moreover, we deduce that the gauge function in the definition of the Henstock-Kurzweil integral can be chosen to be measurable, and an indefinite Henstock-Kurzweil integral generates a sequence of uniformly absolutely continuous finite variational measures (Corollary 3.9).

\section{Preliminaries}

Unless stated otherwise, the following conventions and notations will be used. The set of all real numbers is denoted by $\mathbb{R}$, and the ambient space of this paper is $\mathbb{R}$ with its usual norm. For $x \in \mathbb{R}$ and $r>0$, the open ball $B(x, r)$ is the open interval centered at $x$ with sides equal to $2 r$. For a set $Z \subset \mathbb{R}$, we denote by $\chi_{Z}, \operatorname{int}(Z), \bar{Z}$ and $\operatorname{diam}(Z)$ the characteristic function, interior, closure and diameter of $Z$, respectively. The expressions "absolutely continuous", "measure", "measurable" refer to the one-dimensional Lebesgue measure $\mu_{1}$. A set $Z \subset \mathbb{R}$ is called negligible whenever $\mu_{1}(Z)=0$. Given two subsets $X, Y$ of $\mathbb{R}$, we say that $X$ and $Y$ are nonoverlapping if their intersection is negligible. A function is always real-valued. When no confusion is possible, we do not distinguish between a function defined on a set $Z$ and its restriction to a set $W \subset Z$. If $Z$ is a measurable subset of $\mathbb{R}, \mathcal{L}(Z)$ will denote the space of all Lebesgue integrable functions on $Z$. If $f \in \mathcal{L}(Z)$, the Lebesgue integral of $f$ over $Z$ will be denoted by $(L) \int_{Z} f$.

An interval is a compact nondegenerate interval of $\mathbb{R}$, and $[a, b]$ is a fixed interval. $\mathcal{I}$ is the family of all nondegenerate subintervals of $[a, b]$. If $I \in \mathcal{I}$, we shall write $\mu_{1}(I)$ as $|I|$. A function $F$ defined on $\mathcal{I}$ is said to be additive if $F(I \cup J)=F(I)+F(J)$ for each nonoverlapping intervals $I, J \in \mathcal{I}$ with $I \cup J \in \mathcal{I}$.

A partition is a collection $P=\left\{\left(I_{i}, \xi_{i}\right)\right\}_{i=1}^{p}$, where $I_{1}, I_{2}, \ldots, I_{p}$ are nonoverlapping intervals, and $\xi_{i} \in I_{i}$ for $i=1,2, \ldots, p$. Given $Z \subseteq[a, b]$, a positive function $\delta$ on $Z$ is called a gauge on $Z$. We say that a partition is

(i) a partition in $Z$ if $\bigcup_{i=1}^{p} I_{i} \subset Z$;

(ii) a partition of $Z$ if $\bigcup_{i=1}^{p} I_{i}=Z$;

(iii) anchored in $Z$ if $\left\{\xi_{1}, \xi_{2}, \ldots, \xi_{p}\right\} \subset Z$;

(iv) $\delta$-fine if $I_{i} \subset B\left(\xi_{i}, \delta\left(\xi_{i}\right)\right)$ for each $i=1,2, \ldots, p$. 
In view of the Cousin's lemma [7, Theorem 2.3.1], the following definition is meaningful.

Definition 2.1. A function $f:[a, b] \longrightarrow \mathbb{R}$ is said to be Henstock-Kurzweil integrable if there exists $A \in \mathbb{R}$ such that for any given $\epsilon>0$, there exists a gauge $\delta$ on $[a, b]$ such that

$$
\left|\sum_{i=1}^{p} f\left(\xi_{i}\right)\right| I_{i}|-A|<\epsilon
$$

for each $\delta$-fine partition $\left\{\left(I_{i}, \xi_{i}\right)\right\}_{i=1}^{p}$ of $[a, b]$. Here $A$ is called the HenstockKurzweil integral of $f$ over $[a, b]$, and we write $A=(H K) \int_{a}^{b} f$. If $[a, b]=E$, we also write $A$ as $(H K) \int_{E} f$.

Remark 2.2. (a) The linear space of all Henstock-Kurzweil integrable functions on $[a, b]$ is denoted by $\mathcal{H} \mathcal{K}([a, b])$.

(b) It follows from [7, Theorem 2.5.14] that if $f \in \mathcal{H} \mathcal{K}([a, b])$, then $f \in \mathcal{H} \mathcal{K}(J)$ for each subinterval $J$ of $[a, b]$. The interval function $F: J \mapsto(H K) \int_{J} f$ is known as the indefinite Henstock-Kurzweil integral, or in short the indefinite $\mathcal{H} \mathcal{K}$-integral, of $f$. By [7, Theorem 2.5.12], $F$ is an additive interval function on $\mathcal{I}$.

(c) By [7, Theorem 3.13.3], we see that $\mathcal{L}([a, b]) \subset \mathcal{H K}([a, b])$. Furthermore, $(L) \int_{a}^{b} f=(H K) \int_{a}^{b} f$ for each $f \in \mathcal{L}([a, b])$.

(d) If $f$ is a nonnegative, Henstock-Kurzweil integrable on $[a, b]$, then it follows from $[7$, Theorem 3.13.3] that $f \in \mathcal{L}([a, b])$.

We have the following important Saks-Henstock Lemma [7, Theorem 3.2.1].

Theorem 2.3. (Saks-Henstock). If $F$ is the indefinite $\mathcal{H} \mathcal{K}$-integral of a function $f$ on $[a, b]$, then for $\epsilon>0$, there exists a gauge $\delta$ on $[a, b]$ such that for any $\delta$-fine partition $\left\{\left(I_{i}, \xi_{i}\right)\right\}_{i=1}^{p}$ in $[a, b]$, we have $\sum_{i=1}^{p}\left|f\left(\xi_{i}\right)\right| I_{i}\left|-F\left(I_{i}\right)\right|<\epsilon$.

If $F$ is the indefinite $\mathcal{H} \mathcal{K}$-integral of a function $f$ on $[a, b]$, then it follows from Saks-Henstock Lemma and [7, p.81-82] that $F$ is continuous in the sense that $F(I) \rightarrow 0$ as the measure of the interval $I$ tends to zero. Thus, the space $\mathcal{H} \mathcal{K}([a, b])$ of all Henstock-Kurzweil integrable functions on $[a, b]$ may 
be equipped with the Alexiewicz norm $\|\cdot\|$, where $\|f\|=\sup \left\{\left|(H K) \int_{I} f\right|\right\}$ where the supremum is taken over all subintervals $I$ of $[a, b]$.

Denoting the ordinary derivative of $F$ at $x \in[a, b]$ by $F^{\prime}(x)$, an application of the Vitali covering theorem [13, p.109] that $F^{\prime}(x)$ exists for almost all $x \in[a, b]$ with $F^{\prime}=f$ almost everywhere. In particular, the measurability of $f$ follows easily.

Let $F$ be an interval function on $\mathcal{I}$, and $X$ be an arbitrary subset of $[a, b]$. If $\delta$ is a gauge on $X$, we set $V(F, X, \delta):=\sup _{P} \sum_{i=1}^{p}\left|F\left(I_{i}\right)\right|$ where the supremum is taken over all $\delta$-fine partitions $P=\left\{\left(I_{i}, \xi_{i}\right)\right\}_{i=1}^{p}$ anchored in $X$.

We put $V_{\mathcal{H} K} F(X):=\inf _{\delta} V(F, X, \delta)$ where the infimum is taken over all gauges $\delta$ on $X$. Then, the extended real-valued set function $V_{\mathcal{H} K} F(\cdot)$ has the property that $V_{\mathcal{H} \mathcal{K}} F$ is a metric outer measure. See, for example, [14].

The following Radon-Nikodym Theorem holds for the Henstock-Kurzweil integral.

Theorem 2.4. If $f \in \mathcal{H} \mathcal{K}([a, b])$ with $F$ being its indefinite $\mathcal{H} \mathcal{K}$ integral, then

$$
V_{\mathcal{H} \mathcal{K}} F(X)=(L) \int_{X}|f|
$$

for each measurable subset $X$ of $[a, b]$.

Proof. This follows from [12, Theorem 8] and [12, Proposition 10].

\section{Main Results}

The first theorem is essentially a reformulation of [6, Lemma 15.5] and [6, Lemma 6.18], whose proofs depend on the theory of Denjoy-Perron integration. We shall prove it without reference to the theory of Denjoy-Perron integration [13].

Theorem 3.1. If $f \in \mathcal{H} \mathcal{K}([a, b])$, then there exists an increasing sequence $\left\{Y_{n}\right\}$ of closed sets that satisfies the following conditions:

(a) $\bigcup_{n=1}^{\infty} Y_{n}=[a, b]$;

(b) $f \in \mathcal{L}\left(Y_{n}\right)$ for each $n \in \mathbb{Z}^{+}$;

(c) the series $\sum_{k=1}^{\infty}\left\|f \chi_{\left[c_{k}^{(n)}, d_{k}^{(n)}\right]}\right\|$ converges, where $\left\{\left[c_{k}^{(n)}, d_{k}^{(n)}\right]\right\}$ is the collection of subintervals of $[a, b]$ contiguous to $Y_{n}$. 
Proof. Let $F$ denotes the indefinite Henstock-Kurzweil integral of $f$ on $[a, b]$. By Theorem 2.3, for $\epsilon=1$, there exists a gauge $\delta$ on $[a, b]$ such that for any $\delta$-fine partition $\left\{\left(I_{i}, \xi_{i}\right)\right\}_{i=1}^{p}$ in $[a, b]$, we have

$$
\sum_{i=1}^{p}\left|f\left(\xi_{i}\right)\right| I_{i}\left|-F\left(I_{i}\right)\right|<1 .
$$

For each positive integer $n$, we put

$$
Y_{n}=\overline{\left\{x \in[a, b]:|f(x)|<n \text { and } \delta(x)>\frac{1}{n}\right\}} .
$$

Since $f$ is real-valued and $\delta$ is strictly positive on $[a, b]$, it is clear that (a) holds.

In order to prove (b), given any positive integer $n$, we choose a $\frac{1}{n}$-fine partition $\left\{\left(\left[u_{i}, v_{i}\right], \xi_{i}\right)\right\}_{i=1}^{p}$ anchored in $Y_{n}$. We distinguish the following cases:

(i) If $\xi_{i} \in\left(u_{i}, v_{i}\right)$ or $\xi_{i}=u_{i}=a$ or $\xi_{i}=v_{i}=b$ for some $i \in\{1,2, \ldots, p\}$, we choose $x_{i} \in\left\{x \in[a, b]:|f(x)|<n\right.$ and $\left.\delta(x)>\frac{1}{n}\right\} \cap\left(u_{i}, v_{i}\right)$;

(ii) If $a<u_{i}=\xi_{i}$ for some $i \in\{1,2, \ldots, p\}$, we choose $y_{i} \in\left(u_{i}, v_{i}\right)$ and $x_{i} \in\left\{x \in[a, b]:|f(x)|<n\right.$ and $\left.\delta(x)>\frac{1}{n}\right\}$ so that $u_{i} \in\left(x_{i}, y_{i}\right)$ and the sequence of intervals $\left\{\left[x_{i}, y_{i}\right]\right\}_{i=1}^{p}$ are pairwise disjoint with

$$
\left|F\left(\left[u_{i}, v_{i}\right]\right)-F\left(\left[x_{i}, y_{i}\right]\right)\right|<\frac{1}{p} .
$$

(iii) If $v_{i}=\xi_{i}<b$ for some $i=1,2, \ldots, p$, we choose $x_{i} \in\left(u_{i}, v_{i}\right)$ and $y_{i} \in\left\{x \in[a, b]:|f(x)|<n\right.$ and $\left.\delta(x)>\frac{1}{n}\right\}$ so that $v_{i} \in\left(x_{i}, y_{i}\right)$ and the sequence of intervals $\left\{\left[x_{i}, y_{i}\right]\right\}_{i=1}^{p}$ are pairwise disjoint with

$$
\left|F\left(\left[u_{i}, v_{i}\right]\right)-F\left(\left[x_{i}, y_{i}\right]\right)\right|<\frac{1}{p} .
$$

Put

$$
\begin{aligned}
& T_{1}=\left\{i \in\{1,2, \ldots, p\}: \xi_{i} \in\left(u_{i}, v_{i}\right) \text { or } \xi_{i}=u_{i}=a \text { or } \xi_{i}=v_{i}=b\right\} . \\
& T_{2}=\left\{i \in\{1,2, \ldots, p\}: a<u_{i}=\xi_{i}\right\} . \\
& T_{3}=\left\{i \in\{1,2, \ldots, p\}: v_{i}=\xi_{i}<b\right\} .
\end{aligned}
$$


Then it follows from (i), (ii), (iii) and (3.1) that

$$
\begin{aligned}
& \sum_{i=1}^{p}\left|F\left(\left[u_{i}, v_{i}\right]\right)\right|=\sum_{j=1}^{3} \sum_{i \in T_{j}}\left|F\left(\left[u_{i}, v_{i}\right]\right)\right| \\
< & \sum_{i \in T_{1}}\left|F\left(\left[u_{i}, v_{i}\right]\right)\right|+\sum_{i \in T_{2}}\left|F\left(\left[x_{i}, y_{i}\right]\right)\right|+1+\sum_{i \in T_{3}}\left|F\left(\left[x_{i}, y_{i}\right]\right)\right|+1 \\
< & n(b-a)+1+n(b-a)+1+1+n(b-a)+1+1
\end{aligned}
$$

proving that

$$
V\left(F, Y_{n}, \frac{1}{n}\right) \leq 3 n(b-a)+5 .
$$

Since $V_{\mathcal{H} K} F\left(Y_{n}\right) \leq V\left(F, Y_{n}, \frac{1}{n}\right)$, (b) follows from (3.2) and Theorem 2.4.

In order to prove (c), it suffices to observe that there exists a positive integer $N$ such that

$$
\sum_{k=N}^{\infty}\left(d_{k}^{(n)}-c_{k}^{(n)}\right)<1 \text { and } \sum_{k=N}^{\infty}\left\|f \chi_{\left[c_{k}^{(n)}, d_{k}^{(n)}\right]}\right\| \leq 2 V\left(F, Y_{n}, \frac{1}{n}\right)<\infty .
$$

The next theorem is the Harnack extension for the Henstock-Kurzweil integral.

Theorem 3.2. [2, Theorem 9.22] Let $X$ be a closed subset of $[a, b]$ with $\left\{\left[c_{k}, d_{k}\right]\right\}$ being the collection of subintervals of $[a, b]$ contiguous to $X$. Suppose the following conditions are satisfied:

(a) $f \chi_{X} \in \mathcal{H} \mathcal{K}([a, b])$;

(b) $f \in \mathcal{H} \mathcal{K}\left(\left[c_{k}, d_{k}\right]\right)$ for each positive integer $k$;

(c) the series $\sum_{k=1}^{\infty}\left\|f \chi_{\left[c_{k}, d_{k}\right]}\right\|$ converges;

then $f \in \mathcal{H} \mathcal{K}([a, b])$ and the equality

$$
(H K) \int_{c}^{d} f=(H K) \int_{c}^{d} f \chi_{X}+\sum_{k=1}^{\infty}(H K) \int_{c_{k}}^{d_{k}} f \chi_{[c, d]}
$$

holds for each subinterval $[c, d]$ of $[a, b]$. 
Lemma 3.3. Let $X$ be a closed subset of $[a, b]$ with $\left\{\left[c_{k}, d_{k}\right]\right\}$ being the collection of subintervals of $[a, b]$ contiguous to $X$. Suppose the following conditions are satisfied:

(i) $f \in \mathcal{H} \mathcal{K}([a, b])$;

(ii) $f \chi_{X} \in \mathcal{H} \mathcal{K}([a, b])$;

(iii) the series $\sum_{k=1}^{\infty}\left\|f \chi_{\left[c_{k}, d_{k}\right]}\right\|$ converges;

(iv) $\left\{\left[u_{i}, v_{i}\right]\right\}_{i=1}^{q} \subset[a, b]$ is a finite sequence of nonoverlapping intervals satisfying the condition that at least one of the endpoints of each $\left[u_{i}, v_{i}\right]$ belongs to $X$.

Then

$\sum_{i=1}^{q}\left|(H K) \int_{u_{i}}^{v_{i}}\left(f-f \chi_{X}\right)\right| \leq \sum_{k=1}^{N} \sum_{i=1}^{q}\left|(H K) \int_{u_{i}}^{v_{i}} f \chi_{\left[c_{k}, d_{k}\right]}\right|+2 \sum_{k=N+1}^{\infty}\left\|f \chi_{\left[c_{k}, d_{k}\right]}\right\|$

for each $N \in \mathbb{Z}^{+}$.

Proof. By (i), $f \in \mathcal{H} \mathcal{K}\left(\left[c_{k}, d_{k}\right]\right)$ for each positive integer $k$. In view of (ii), (iii) and Theorem 3.2, we have $(H K) \int_{u_{i}}^{v_{i}}\left(f-f \chi_{X}\right)=\sum_{k=1}^{\infty}(H K) \int_{c_{k}}^{d_{k}} f \chi_{\left[u_{i}, v_{i}\right]}$ for each $i=1,2, \ldots, q$. Thus, we have

$$
\begin{aligned}
& \left|(H K) \int_{u_{i}}^{v_{i}}\left(f-f \chi_{X}\right)\right| \\
\leq & \sum_{k=1}^{\infty}\left|(H K) \int_{c_{k}}^{d_{k}} f \chi_{\left[u_{i}, v_{i}\right]}\right| \\
\leq & \sum_{k=1}^{N}\left|(H K) \int_{c_{k}}^{d_{k}} f \chi_{\left[u_{i}, v_{i}\right]}\right|+\sum_{k=N+1}^{\infty}\left|(H K) \int_{c_{k}}^{d_{k}} f \chi_{\left[u_{i}, v_{i}\right]}\right|
\end{aligned}
$$


giving

$$
\begin{aligned}
& \sum_{i=1}^{q}\left|(H K) \int_{u_{i}}^{v_{i}}\left(f-f \chi_{X}\right)\right| \\
\leq & \sum_{k=1}^{N} \sum_{i=1}^{q}\left|(H K) \int_{c_{k}}^{d_{k}} f \chi_{\left[u_{i}, v_{i}\right]}\right|+\sum_{k=N+1}^{\infty} \sum_{i=1}^{q}\left|(H K) \int_{c_{k}}^{d_{k}} f \chi_{\left[u_{i}, v_{i}\right]}\right| \\
\leq & \sum_{k=1}^{N} \sum_{i=1}^{q}\left|(H K) \int_{u_{i}}^{v_{i}} f \chi_{\left[c_{k}, d_{k}\right]}\right|+2 \sum_{k=N+1}^{\infty}\left\|f \chi_{\left[c_{k}, d_{k}\right]}\right\|
\end{aligned}
$$

by (iv), since each interval $\left[c_{k}, d_{k}\right]$ can intersect with at most two intervals belonging to the set $\left\{\left[u_{i}, v_{i}\right]\right\}_{i=1}^{q}$.

Theorem 3.4. Let $X$ be a closed subset of $[a, b]$ with $\left\{\left[c_{k}, d_{k}\right]\right\}$ being the collection of subintervals of $[a, b]$ contiguous to $X$. Suppose the following conditions are satisfied:

(i) $f \in \mathcal{H} \mathcal{K}([a, b])$;

(ii) $f \chi_{X} \in \mathcal{H} \mathcal{K}([a, b])$;

(iii) the series $\sum_{k=1}^{\infty}\left\|f \chi_{\left[c_{k}, d_{k}\right]}\right\|$ converges;

then given $\epsilon>0$, there exists a constant gauge $\delta$ on $X$ such that for any $\delta$-fine partition $\left\{\left(\left[u_{i}, v_{i}\right], \xi_{i}\right)\right\}_{i=1}^{p}$ anchored in $X$, we have

$$
\sum_{i=1}^{p}\left|(H K) \int_{u_{i}}^{v_{i}} f \chi_{X}-(H K) \int_{u_{i}}^{v_{i}} f\right|<\epsilon .
$$

Proof. By (iii), for $\epsilon>0$, there exists $N \in \mathbb{Z}^{+}$such that

$$
\sum_{k=N+1}^{\infty}\left\|f \chi_{\left[c_{k}, d_{k}\right]}\right\|<\frac{\epsilon}{4}
$$

By (i), $f \in \mathcal{H} \mathcal{K}\left(\left[c_{k}, d_{k}\right]\right)$ for each $k$. Since $f \in \mathcal{H} \mathcal{K}\left(\left[c_{i}, d_{i}\right]\right)$ for each $i=$ $1,2, \ldots, N$, it follows from the continuity of indefinite $\mathcal{H} \mathcal{K}$-integral that there exists $\eta_{i}>0$ such that whenever $[u, v] \subseteq\left[c_{i}, d_{i}\right]$ satisfying $v-u<\eta_{i}$, we have

$$
\left|(H K) \int_{u}^{v} f\right|<\frac{\epsilon}{4 N}
$$


Define a constant gauge $\delta$ on $[a, b]$ by $\delta=\min _{i=1,2, \ldots, N} \eta_{i}$. An application of Lemma 3.3, (3.3) and (3.4) shows that for any $\delta$-fine partition $P=\left\{\left(I_{i}, \xi_{i}\right)\right\}_{i=1}^{p}$ anchored in $X$, we have

$$
\begin{aligned}
& \sum_{i=1}^{p}\left|(H K) \int_{u_{i}}^{v_{i}}\left(f-f \chi_{X}\right)\right| \\
\leq & \sum_{k=1}^{N} \sum_{i=1}^{p}\left|(H K) \int_{u_{i}}^{v_{i}} f \chi_{\left[c_{k}, d_{k}\right]}\right|+2 \sum_{k=N+1}^{\infty}\left\|f \chi_{\left[c_{k}, d_{k}\right]}\right\| \\
< & 2 N \frac{\epsilon}{4 N}+2 \frac{\epsilon}{4}=\epsilon .
\end{aligned}
$$

In what follows, we shall write a decreasing null sequence of positive numbers $\left\{\epsilon_{n}\right\}$ as $\epsilon_{n} \downarrow 0$.

Theorem 3.5. If $f \in \mathcal{H} \mathcal{K}([a, b])$, then for $\epsilon_{n} \downarrow 0$, there exists an increasing sequence $\left\{X_{n}\right\}$ of closed sets such that

(i) $\bigcup_{n=1}^{\infty} X_{n}=[a, b]$;

(ii) $f \in \mathcal{L}\left(X_{n}\right)$ for each $n \in \mathbb{Z}^{+}$;

(iii) for each positive integer $n$, there exists a partition $P_{n}=\left\{\left(I_{i}, \xi_{i}\right)\right\}_{i=1}^{p}$ of $[a, b]$ such that the inequality

$$
\sum_{i=1}^{p} \sum_{J \subseteq I_{i}}\left|(L) \int_{J \cap X_{n}} f-(H K) \int_{J} f\right|<\epsilon_{n}
$$

holds whenever $\{J\}$ is a finite sequence of non-overlapping subintervals of $[a, b]$ satisfying $J \cap X_{n} \neq \emptyset$ for all $n$.

Proof. Since $\epsilon_{n} \downarrow 0$, we may assume that $\epsilon_{n}=\frac{1}{n}$. Since $f$ is HenstockKurzweil integrable on $[a, b]$, there exists an increasing sequence $\left\{Y_{k}\right\}$ of closed sets satisfying all the conditions of Theorem 3.1. By Theorem 3.4, for each $k \in \mathbb{Z}^{+}$, there exists a constant gauge $\delta_{k}^{\prime}$ on $Y_{k}$ such that for any $\delta_{k}^{\prime}$-fine partition $\left\{\left(I_{i}, \xi_{i}\right)\right\}_{i=1}^{q}$ anchored in $Y_{k}$, we have

$$
\sum_{i=1}^{q}\left|(L) \int_{I_{i} \cap Y_{k}} f-(H K) \int_{I_{i}} f\right|<\frac{1}{k} .
$$

Next, we want to choose $\left\{X_{n}\right\}$ from $\left\{Y_{k}\right\}$ so that the required properties hold. 
Let $p(n, k)=2^{k} n$. Define a gauge $\delta_{n}$ on $[a, b]$ by

$$
\delta_{n}(\xi)= \begin{cases}\delta_{p(n, 1)^{\prime}}(\xi) & \text { if } \xi \in Y_{p(n, 1)} \\ \left.\min \left\{\delta_{p(n, k)}^{\prime}, \operatorname{dist}\left(\xi, Y_{p(n, k-1}\right)\right)\right\} & \text { if } \xi \in Y_{p(n, k)} \backslash Y_{p(n, k-1)} \\ & \text { for some } k \geq 2\end{cases}
$$

Since $\delta_{n}$-fine partitions of $[a, b]$ exist, we may fix a $\delta_{n}$-fine partition $P_{n}=$ $\left\{\left(I_{i}, \xi_{i}\right)\right\}_{i=1}^{p}$ of $[a, b]$. For simplicity, we put

$$
Q_{1}=Y_{p(n, 1)} \text { and } Q_{k}=Y_{p(n, k)} \backslash Y_{p(n, k-1)} \text { for } k \geq 2
$$

Next, we put

$$
X_{n}=\bigcup_{k=1}^{\infty}\left\{I \cap Y_{p(n, k)}:(I, \xi) \in P_{n} \text { with } \xi \in Q_{k}\right\} .
$$

The above union is a finite one because $P_{n}$ only has finitely many terms. Thus $X_{n}$ is closed as each $Y_{k}$ is closed.

Define $k(n)=\max \left\{k:(I, \xi) \in P_{n}\right.$ and $\left.\xi \in Q_{k}\right\}$. Since $\left\{Y_{k}\right\}$ is an increasing sequence of closed sets whose union is $[a, b]$, we have $Y_{p(n, k(n))} \supseteq X_{n}$. By the definition of $\delta_{n}$ and the compactness of $Y_{p(n, 1)}$, the $\delta_{n}$-fine partition $P_{n}=\left\{\left(I_{i}, \xi_{i}\right)\right\}_{i=1}^{p}$ must cover $Y_{p(n, 1)}$. Hence $Y_{p(n, 1)} \subseteq X_{n}$. Thus, we have $Y_{p(n, 1)} \subseteq X_{n} \subseteq Y_{p(n, k(n))}$ and $f \in \mathcal{L}\left(X_{n}\right)$ because $X_{n}$ is measurable. Observe also that if $(I, \xi) \in P_{n}$ with $\xi \in Q_{k}$ for some positive integer $k$, then $I \cap$ $X_{n}=I \cap Y_{p(n, k)}$. Note that each $(I, \xi) \in P_{n}$ may have its associated points $\xi$ belonging to $Q_{1}$ only. Without loss of generality, we may suppose that each $(I, \xi) \in P_{n}$ has its associated point $\xi$ belongs to $Q_{s_{1}}, Q_{s_{2}}, \ldots, Q_{s_{l}}$ for some positive integers $s_{1}<s_{2}<\cdots<s_{l}$ with $s_{1}=1$. Let $\{J\}$ be a finite sequence of non-overlapping subintervals of $[a, b]$ with $J \subseteq I_{i}$ for some $i=1,2, \ldots, p$, 
and $J \cap X_{n} \neq \emptyset$. Then we have

$$
\begin{aligned}
& \sum_{i=1}^{p} \sum_{J \subseteq I_{i}}\left|(L) \int_{J \cap X_{n}} f-(H K) \int_{J} f\right| \\
= & \sum_{i=1}^{p} \sum_{k=1}^{l} \sum_{J \subseteq I_{i}: \xi_{i} \in I_{i} \cap Q_{s_{k}}}\left|(L) \int_{J \cap X_{n}} f-(H K) \int_{J} f\right| \\
= & \sum_{i=1}^{p} \sum_{k=1}^{l} \sum_{J \subseteq I_{i}: \xi_{i} \in I_{i} \cap Q_{s_{k}}}\left|(L) \int_{J \cap Y_{p\left(n, s_{k}\right)}} f-(H K) \int_{J} f\right| \\
= & \sum_{k=1}^{l} \sum_{i=1}^{p} \sum_{J \subseteq I_{i}: \xi_{i} \in I_{i} \cap Q_{s_{k}}}\left|(L) \int_{J \cap Y_{p\left(n, s_{k}\right)}} f-(H K) \int_{J} f\right| \\
< & \sum_{k=1}^{l} \frac{1}{n 2^{s_{k}}}<\frac{1}{n} .
\end{aligned}
$$

It is easy to see that there exists an increasing subsequence of $\left\{X_{n}\right\}$, denoted again by $\left\{X_{n}\right\}$, such that $\bigcup_{n=1}^{\infty} X_{n}=[a, b]$.

Corollary 3.6. $[10$, Theorem 2] If $f \in \mathcal{H} \mathcal{K}([a, b])$, then the following condition is satisfied: Given $\epsilon_{n} \downarrow 0$, there exists a sequence $\left\{X_{n}\right\}$ of closed sets in $[a, b]$ such that:

(i) $a, b \in X_{1}, X_{n} \subseteq X_{n+1}$ for all $n$ and $\bigcup_{n=1}^{\infty} X_{n}=[a, b]$;

(ii) $f \in \mathcal{L}\left(X_{n}\right)$ for each $n$;

(iii) for each positive integer $n$, if a finite sequence $\left\{I_{i}\right\}_{i=1}^{q}$ of non-overlapping intervals contained in $[a, b]$ satisfies the condition that at least one of the endpoints of each $I_{i}$ belong to $X_{n}$, then we have

$$
\sum_{i=1}^{q}\left|(L) \int_{I_{i} \cap X_{n}} f-(H K) \int_{I_{i}} f\right|<\epsilon_{n} .
$$

Theorem 3.7. If $f \in \mathcal{H} \mathcal{K}([a, b])$, then there exists an increasing sequence $\left\{X_{n}\right\}$ of closed sets whose union is $[a, b],\left\{f \chi_{X_{n}}\right\} \subset \mathcal{L}([a, b])$ and $\left\{f \chi_{X_{n}}\right\}$ satisfies the following conditions:

(i) $f \chi_{X_{n}} \rightarrow f$ everywhere on $[a, b]$; 
(ii) for $\epsilon>0$, there exists a measurable gauge $\delta$, independent of $n$, on $[a, b]$ such that for every $\delta$-fine partition $P=\left\{\left(I_{i}, \xi_{i}\right)\right\}_{i=1}^{p}$ of $[a, b]$, we have

$$
\left|\sum_{i=1}^{p} f\left(\xi_{i}\right) \chi_{X_{n}}\left(\xi_{i}\right)\right| I_{i}\left|-(L) \int_{a}^{b} f \chi_{X_{n}}\right|<\epsilon
$$

for all $n \in \mathbb{Z}^{+}$. In particular, $\left\{f \chi_{X_{n}}\right\}$ is Henstock-Kurzweil equi-integrable on $E$.

Proof. By Corollary 3.6, we choose $\left\{X_{k}\right\}$ corresponding to $\epsilon_{k}=\frac{1}{k^{2}}$ and put $f_{k}=f \chi_{x_{k}}$ for $k \in \mathbb{Z}^{+}$. An application of [11, Proposition 4] and [11, Lemma 7 (iii)] shows that for $\epsilon>0$, there exists a measurable gauge $\delta_{k}$ on $[a, b]$ such that for any $\delta_{k}$-fine partition $P_{1}=\left\{\left(I_{i}, \xi_{i}\right)\right\}_{i=1}^{p_{1}}$ in $[a, b]$, we have

$$
\sum_{i=1}^{p_{1}}\left|f_{k}\left(\xi_{i}\right)\right| I_{i}\left|-\int_{I_{i}} f_{k}\right|<\frac{\epsilon}{2^{k+2}} .
$$

We may also assume that for each $x \in[a, b]$, the sequence $\left\{\delta_{k}(x)\right\}$ is nonincreasing. Choose a positive integer $N \geq 2$ such that

$$
\sum_{k=N}^{\infty} \frac{1}{k^{2}}<\frac{\epsilon}{4}
$$

Let $\left\{\left[c_{i}^{(N)}, d_{i}^{(N)}\right]\right\}$ be the sequence of subintervals of $[a, b]$ contiguous to $X_{N}$ and put $\eta=\frac{1}{4} \min _{1 \leq i \leq N}\left|d_{i}^{(N)}-c_{i}^{(N)}\right|$.

Define a gauge $\delta$ on $[a, b]$ by

$\delta(\xi)= \begin{cases}\min \left\{\delta_{N}(\xi), \eta\right\} & \text { if } \xi \in X_{1}, \\ \min \left\{\delta_{N}(\xi), \eta\right\} & \text { if } \xi \in X_{1}, \\ \min \left\{\delta_{N}(\xi), \operatorname{dist}\left(\xi, X_{k-1},\right), \eta\right\} & \text { if } \xi \in X_{k} \backslash X_{k-1} \text { for some } 2 \leq k \leq N \\ \min \left\{\delta_{k}(\xi), \operatorname{dist}\left(\xi, X_{k-1}\right), \eta\right\} & \text { if } \xi \in X_{k} \backslash X_{k-1} \text { for some } k>N .\end{cases}$

Then $\delta$ is a measurable gauge on $[a, b]$ with

$$
\delta(\xi) \leq \delta_{N}(\xi) \text { for each } \xi \in X_{N}
$$

and

$$
\delta(\xi) \leq \delta_{k}(\xi) \text { if } \xi \in X_{k} \backslash X_{k-1} \text { for some } k>N .
$$


Claim. The sequence $\left\{f_{n}\right\}$ is Henstock-Kurzweil equi-integrable with this function $\delta$. Given a $\delta$-fine partition $P=\left\{\left(I_{i}, \xi_{i}\right)\right\}_{i=1}^{p}$ of $[a, b]$, we put

$$
N_{0}=\max \left\{i:(I, \xi) \in P \text { with } \xi \in X_{i} \cdot\right\} .
$$

Then by our definition of $\eta$ and $\delta$, any $\delta$-fine cover of $X_{N}$ cannot be a cover of $\left[c_{i}^{(N)}, d_{i}^{(N)}\right]$ for $i=1,2,3, \ldots, N$, so we have $N_{0}>N$.

Subclaim 1. $\sum_{i=1}^{p}\left|f\left(\xi_{i}\right)\right| I_{i}\left|-(H K) \int_{I_{i}} f\right|<\frac{\epsilon}{2}$.

Let $S_{N}=\left\{i: \xi_{i} \in X_{N}\right\}$ and $S_{k}=\left\{i: \xi_{i} \in X_{k} \backslash X_{k-1}\right\}$ for each $k>N$. Then it follows from (3.5) to (3.8) that we have

$$
\begin{aligned}
& \sum_{i=1}^{p}\left|f\left(\xi_{i}\right)\right| I_{i}\left|-(H K) \int_{I_{i}} f\right| \\
\leq & \sum_{i \in S_{N}}\left|f\left(\xi_{i}\right)\right| I_{i}\left|-(H K) \int_{I_{i}} f\right|+\sum_{k=N+1}^{N_{0}} \sum_{i \in S_{k}}\left|f\left(\xi_{i}\right)\right| I_{i}\left|-(H K) \int_{I_{i}} f\right| \\
\leq & \sum_{i \in S_{N}}\left|f_{N}\left(\xi_{i}\right)\right| I_{i}\left|-(L) \int_{I_{i}} f_{N}\right|+\sum_{i \in S_{N}}\left|(L) \int_{I_{i}} f_{N}-(H K) \int_{I_{i}} f\right| \\
+ & \sum_{k=N+1}^{N_{0}} \sum_{i \in S_{k}}\left|f_{k}\left(\xi_{i}\right)\right| I_{i}\left|-(L) \int_{I_{i}} f_{k}\right|+\sum_{k=N+1}^{N_{0}} \sum_{i \in S_{k}}\left|(L) \int_{I_{i}} f_{k}-(H K) \int_{I_{i}} f\right| \\
< & \frac{\epsilon}{2^{N+2}}+\frac{1}{N^{2}} \\
+ & \sum_{k=N+1}^{N_{0}} \sum_{i \in S_{k}}\left|f_{k}(\xi)\right| I_{i}\left|-(L) \int_{I_{i}} f_{k}\right|+\sum_{k=N+1}^{N_{0}} \sum_{i \in S_{k}}\left|(L) \int_{I_{i}} f_{k}-(H K) \int_{I_{i}} f\right| \\
< & \frac{\epsilon}{2^{N+2}}+\frac{1}{N^{2}}+\sum_{k=N+1}^{N_{0}} \frac{\epsilon}{2^{k+2}}+\sum_{k=N+1}^{N_{0}} \frac{1}{k^{2}}<\frac{\epsilon}{2} .
\end{aligned}
$$

The next two subclaims will enable us to prove that $\left\{f_{n}\right\}$ is HenstockKurzweil equi-integrable on $[a, b]$.

Subclaim 2. For each $n=1,2, \ldots, N$, we have

$$
\sum_{i=1}^{p}\left|f_{n}\left(\xi_{i}\right)\right| I_{i}\left|-(L) \int_{I_{i}} f_{n}\right|<\frac{\epsilon}{2^{n+2}} .
$$


By our definition of $\delta,(\xi-\delta(\xi), \xi+\delta(\xi)) \subset(a, b) \backslash X_{n}$ whenever $\xi \notin X_{n}$, so

$$
\begin{aligned}
& \sum_{i=1}^{p}\left|f_{n}\left(\xi_{i}\right)\right| I_{i}\left|-(L) \int_{I_{i}} f_{n}\right| \\
= & \sum_{i: \xi_{i} \in X_{n}}\left|f_{n}\left(\xi_{i}\right)\right| I_{i}\left|-(L) \int_{I_{i}} f_{n}\right|+\sum_{i: \xi_{i} \notin X_{n}}\left|f_{n}\left(\xi_{i}\right)\right| I_{i}\left|-(L) \int_{I_{i}} f_{n}\right| \\
= & \sum_{i: \xi_{i} \in X_{n}}\left|f_{n}\left(\xi_{i}\right)\right| I_{i}\left|-(L) \int_{I_{i}} f_{n}\right|<\frac{\epsilon}{2^{n+2}} .
\end{aligned}
$$

Subclaim 3. For each integer $n$ with $n>N, \sum_{i=1}^{p}\left|f_{n}\left(\xi_{i}\right)\right| I_{i}\left|-\int_{I_{i}} f_{n}\right|<\epsilon$. Since $f=f_{n}$ on $X_{n}$, we have

$$
\begin{aligned}
& \sum_{i=1}^{p}\left|f_{n}\left(\xi_{i}\right)\right| I_{i}\left|-(L) \int_{I_{i}} f_{n}\right| \\
= & \sum_{i: \xi_{i} \in X_{n}}\left|f_{n}\left(\xi_{i}\right)\right| I_{i}\left|-(L) \int_{I_{i}} f_{n}\right|+\sum_{i: \xi_{i} \notin X_{n}}\left|f_{n}\left(\xi_{i}\right)\right| I_{i}\left|-(L) \int_{I_{i}} f_{n}\right| \\
= & \sum_{i: \xi_{i} \in X_{n}}\left|f_{n}\left(\xi_{i}\right)\right| I_{i}\left|-(L) \int_{I_{i}} f_{n}\right| \\
= & \sum_{i: \xi_{i} \in X_{n}}\left|f\left(\xi_{i}\right)\right| I_{i}\left|-(L) \int_{I_{i}} f_{n}\right| \\
\leq & \sum_{i: \xi_{i} \in X_{n}}\left|f\left(\xi_{i}\right)\right| I_{i}\left|-(H K) \int_{I_{i}} f\right|+\sum_{i: \xi_{i} \in X_{n}}\left|(L) \int_{I_{i}} f_{n}-(H K) \int_{I_{i}} f\right| \\
< & \frac{\epsilon}{2}+\frac{1}{n^{2}}<\frac{\epsilon}{2}+\sum_{k=N}^{\infty} \frac{1}{k^{2}}<\epsilon .
\end{aligned}
$$

From subclaims 2 and 3, we have, for all positive integer $n$,

$$
\left|\sum_{i=1}^{p} f_{n}\left(\xi_{i}\right)\right| I_{i}\left|-(L) \int_{a}^{b} f_{n}\right|<\epsilon .
$$

From the subclaim 1 of the proof of Theorem 3.7 and the measurability of the $\delta$ function, we obtain the following corollary, which was proved differently in [6, Theorem 10.3] or [2, Theorem 9.24]. 
Corollary 3.8 If $f \in \mathcal{H} \mathcal{K}([a, b])$, then for $\epsilon>0$, the function $\delta$ from the definition of the Henstock-Kurzweil integral can be chosen to be measurable.

Corollary 3.9 If $f \in \mathcal{H} \mathcal{K}([a, b])$ with $F$ being its indefinite $\mathcal{H} \mathcal{K}$ integral, then there exists a sequence $\left\{F_{n}\right\}$ of additive interval functions on $\mathcal{I}$ satisfying the following conditions:

(i) $V_{\mathcal{H} K} F_{n}([a, b])<\infty$ for each $n$;

(ii) given that $Z \subset E$ is negligible and $\epsilon>0$, there exists a gauge $\delta$, independent of $n$, on $Z$ such that

$$
V\left(F_{n}, Z, \delta\right)<\epsilon \text { for all } n \text {. }
$$

We remark that the proofs of Corollary 3.6, Theorem 3.7 and Corollary 3.9 do not generalize to the higher dimensional interval $E:=\left[a_{1}, b_{1}\right] \times\left[a_{2}, b_{2}\right] \times$ $\cdots \times\left[a_{m}, b_{m}\right]$ of $\mathbb{R}^{m}$. Indeed, the proof is based on Theorem 3.4 , for which no satisfactory analogue in higher dimensions is known. As a result, we have the following conjecture.

Conjecture 1. If the sequence of additive interval functions $\left\{F_{n}\right\}$ satisfies the following condition:

Given that $Z \subset E$ is negligible and $\epsilon>0$, there exists a gauge $\delta$, independent of $n$, on $Z$ such that

$$
V\left(F_{n}, Z, \delta\right)<\epsilon \text { for all } n
$$

then there exists a sequence of functions $\left\{f_{n}\right\}$ on $E$ satisfying the following conditions:

(i) for each $n=1,2, \ldots, F_{n}$ is the indefinite $\mathcal{H} \mathcal{K}$-integral of $f_{n}$ on $E$;

(ii) $\left\{f_{n}\right\}$ is Henstock-Kurzweil equi-integrable on $E$.

If we assume that $F_{n} \equiv F$ for all $n$, then conjecture 1 turns out to be true. This result was obtained for $m=1$ in [1], and for $m \geq 1$ in [8].

The proof of Corollary 3.6 is real-line dependent. As a result, it is natural to ask whether the next conjecture is true for $m \geq 2$.

Conjecture 2. If $f \in \mathcal{H} \mathcal{K}(E)$, then given $\epsilon_{n} \downarrow 0$, there exists an increasing sequence $\left\{X_{n}\right\}$ of closed sets satisfying the following conditions:

(i) $\bigcup_{n=1}^{\infty} X_{n}=E$; 
(ii) $f \in \mathcal{L}\left(X_{n}\right)$ for each $n$;

(iii) for each $n$, there exists a positive constant $\eta_{n}$ such that whenever $\left\{\left(I_{i}, \xi_{i}\right)\right\}_{i=1}^{p}$ is a $\eta_{n}$-fine partition anchored in $X_{n}$, we have

$$
\sum_{i=1}^{p}\left|(L) \int_{I_{i} \cap X_{n}} f-(H K) \int_{I_{i}} f\right|<\epsilon_{n} .
$$

Since the proof of Theorem 3.7 depends on Corollary 3.6, it is also natural to ask whether the following analogue of Theorem 3.7 holds if $m \geq 2$.

Conjecture 3. If $f \in \mathcal{H} \mathcal{K}(E)$, then there exists an increasing sequence $\left\{X_{n}\right\}$ of closed sets $\left\{X_{n}\right\}$ satisfying the following conditions:

(i) $X_{n} \subseteq E$ for all $n$;

(ii) $Z:=E \backslash \bigcup_{k=1}^{\infty} X_{k}$ has $m$-dimensional Lebesgue measure zero;

(iii) $f \in \mathcal{L}\left(X_{n}\right)$ for each $n$;

(iv) $\left\{f \chi_{X_{n} \cup Z}\right\}$ is Henstock-Kurzweil equi-integrable on $E$.

\section{References}

[1] B. Bongiorno, L. Di Piazza and V. Skvortsov, A new descriptive characterization of Denjoy-Perron integral, Real Anal. Exchange 21 (1995/96), 656-663.

[2] R. A. Gordon, The integrals of Lebesgue, Denjoy, Perron, and Henstock, Graduate Studies in Mathematics, 4, American Mathematical Society, Providence, RI, 1994.

[3] J. Kurzweil, Henstock-Kurzweil integration, its relation to topological vector spaces, Series in Real Analysis Volume, 7, World Scientific 2000.

[4] J. Kurzweil and J. Jarník, Equiintegrability and Controlled Convergence of Perron-type integrable functions, Real Anal. Exchange, 17 (1991/92), 110-139.

[5] Lee Peng Yee and Chew Tuan Seng, A Riesz-type definition of the Denjoy integral, Real Anal. Exchange, 11 (1985/86), 221-227.

[6] Lee Peng Yee, Lanzhou Lectures on Henstock integration, Series in Real Analysis, 2, World Scientific 1989. 
[7] Lee Peng Yee and Rudolf Výborný, The integral, An Easy Approach after Kurzweil and Henstock, Australian Mathematical Society Lecture Series, 14, Cambridge University Press 2000.

[8] Lee Tuo Yeong, A full descriptive definition of the Henstock-Kurzweil integral in the Euclidean space, Proc. London Math. Soc. to appear.

[9] E. J. McShane, Integration, Princeton University Press, 1944.

[10] S Nakanishi, A new definition of the Denjoy's special integral by the method of successive approximation, Math. Japonica 41, No. 1 (1995), $217-230$.

[11] W. F. Pfeffer, A note on the Generalized Riemann integral, Proc. Amer. Math. Soc., 103, No. 4 (1988), 1161-1166.

[12] W. F. Pfeffer, The Lebesgue and Denjoy-Perron integrals from a descriptive point of view, Ricerche Mat. 48 (1999), no. 2, 211-223.

[13] S. Saks, Theory of the integral, 2nd edn, New York, 1964.

[14] B. S. Thomson, Derivates of Interval Functions, Mem. Amer. Math. Soc. 452, Providence, 1991. 